(2) Open Access Full Text Article

REVIEW

\title{
Ultradeformable phospholipid vesicles as a drug delivery system: a review
}

Maria Jose Morilla

Eder Lilia Romero

Nanomedicine Research Program, Department of Science and Technology, National University of Quilmes, Buenos Aires, Argentina
Correspondence: Eder Lilia Romero Programa de Nanomedicinas,

Departamento de Ciencia y Tecnología,

Universidad Nacional de Quilmes, Roque

Saenz Peña 352, Bernal, BI876 BXD,

Buenos Aires, Argentina

Tel +54 II 43657100

Fax +54 I| 4365 7। 32

Email elromero@unq.edu.ar
This article was published in the following Dove Press journal:

Research and Reports in Transdermal Drug Delivery

I December 2015

Number of times this article has been viewed

\begin{abstract}
Ultradeformable vesicles are highly deformable (elastic/flexible) liposomes made of phospholipids plus highly mobile hydrophilic detergents capable of penetrating the intact skin across the stratum corneum and reaching the viable epidermis. Ultradeformable vesicles are more effective than conventional liposomes in delivering drugs into and across the mammalian skin, and these have been tested for local delivery of nonsteroidal anti-inflammatory agents against musculoskeletal-articular disorders such as osteoarthritis, to treat atopic dermatitis, to deliver anti-infective drugs against epithelial infections, and to avoid the first-pass effect of oral antihypertensive drugs. Currently, a single ultradeformable vesicle anti-inflammatory formulation has reached Phase III clinical trials, but no commercial product based on this technology is yet available.
\end{abstract}

Keywords: topical administration, skin penetration, antihypertensive drugs, anti-leishmanial drugs, anti-infective drugs

\section{Introduction}

The three principal targets for topical and transdermal drug delivery are the skin surface, the skin itself (epidermis or dermis), and the subcutaneous tissue or systemic circulation. ${ }^{1}$ The skin surface is a target for disinfectants, insect repellents, or cosmetics. Targeting the various layers of the skin becomes relevant when the disease state presents within the organ itself, eg, neoplasias, inflammatory disorders, and microbial infections. In this case, the topical route provides direct accessibility to the targets located a few microns under the surface, enabling increased biological potency and prolonged effect, thereby reducing therapeutic dosage and the risk of adverse events as compared with parenteral administration. Transdermal or percutaneous delivery, in which the subcutaneous tissue or systemic circulation is the principal target, is an alternative to systemic and oral routes of administration. The transdermal route offers numerous advantages relative to the oral route, including avoidance of degradation by the harsh gastrointestinal (GI) medium, avoidance of systemic first-pass metabolism, and high patient compliance.

The epidermis is the outermost layer of the skin, having thickness ranging from $50 \mu \mathrm{m}$ in the eyelids to $200 \mu \mathrm{m}$ in the sole and palms. It is a stratifed squamous epithelium composed of - from the surface to the inner layers - the stratum corneum (SC, horny layer), stratum granulosum (granular cell layer), stratum spinosum (spinous or prickle cell layer), and stratum basale (basal or germinativum cell layer, mainly dividing and nondividing keratinocytes, which are attached to the basement membrane by hemidesmosomes). In addition, the stratum lucidum is a thin layer of translucent cells seen in the thick epidermis. It represents a transition from the stratum granulosum and 
SC and is not usually seen in the thin epidermis. Together, the stratum spinosum and stratum basale are sometimes referred to as the Malpighian layer. Moreover, $90 \%$ of the epidermal cells are keratinocytes, 1\% Langerhans cells, and the remaining are melanocytes and Merkel cells (basal layer). ${ }^{2}$ The SC presents a dry thickness of 10-15 $\mu \mathrm{m}$ and comprises 15-20 corneocyte (flattened, elongated, dead cells) layers rich in keratin joined together by desmosomes and embedded in a nonphospholipid lipid matrix (ceramides, fatty acids, cholesterol, cholesterol sulfate, and sterol/wax esters, arranged in multiple bilayers called lamellae). The corneocyte layer can absorb three times its weight in water but if its water content drops to $<10 \%$, it no longer remains pliable and cracks. The movement of epidermal cells to this layer usually takes approximately 28 days and is known as the epidermal transit time. Only small and hydrophobic molecules can penetrate the SC because the penetration of molecules with molecular weight $(\mathrm{MW})>500 \mathrm{Da}$ is impaired at this level. ${ }^{3}$ The barrier properties of the SC have been assigned to the multiple lipid bilayers residing in the intercellular space. These bilayers prevent desiccation of the underlying tissues by inhibiting water loss and limiting the penetration of substances from the external environment. Consequently, there has been a concerted effort to investigate and develop novel strategies to maximize the amount of drugs crossing this layer. One of the most attractive strategies developed to penetrate the $\mathrm{SC}$ is the use of soft matter-based particulate drug delivery systems, in particular nanosized drug delivery systems.

The most popular soft matter-based nanosized drug delivery systems are the liposomes, first described in 1964 by Bangham and Horne. ${ }^{4}$ Liposomes are closed micro- or nanovesicles having one or multiple phospholipid bilayers, formed in an excess of water. Engineering advances led to long blood circulation lifetime and high drug payload liposomes, allowed industrial production under good manufacturing practices conditions, and increased liposomal shelf life. As a consequence, ten liposomal therapeutic products and vaccines were released in the global market, and 23 liposomal formulations are in Phase II and Phase III clinical trials. ${ }^{5,6}$ Upon being loaded in liposomes, a drug's pharmacokinetics and biodistribution are modified, and a reduction of its adverse effects is achieved. Therefore, intravenously administered liposomes are mainly used for antitumoral drug delivery. ${ }^{6}$ Liposomes are also used for topical drug delivery, but once on the SC surface, liposomes have been observed to act as drug depots without entering the SC. Accessing deeper epithelial layers, without the aid of permeation enhancers, has been enabled by tuning the elastomechanical properties of liposomal bilayers. At the beginning of the 1990s, the pioneering work of Gregor Cevc produced the so-called ultradeformable vesicles (UDVs, known as Transfersomes ${ }^{\circledR}$ ). The elastic energy barrier for the bilayer surface fluctuations on the UDV has been estimated to be one to two orders of magnitude lower than that for conventional liposomes. ${ }^{7}$ Transfersomes could efficiently penetrate the SC instead of stacking on the first corneocyte layer. ${ }^{8}$ Up to now, however, neither Transfersomes nor any other highly deformable vesicle has been unquestionably shown to penetrate beyond the SC in intact form. ${ }^{9}$ A recent review shows the relative advantages (compared with conventional nondeformable liposomal formulations) of UDVs in the treatment of musculoskeletal diseases such as osteoarthritis and skin diseases such as atopic dermatitis, psoriasis, and nonmelanoma skin cancer. ${ }^{10}$ Today, the problem of accessing deeper epidermal layers without entering the dermis or reaching the systemic circulation remains unsolved. Probably, solely increasing bilayer deformability is insufficient and a more specifically tailored liposomal bilayer, combined with specific drugs and dosage regimens, is required. In the past 2 years, new analytical tools have allowed distinguishing the true pathways followed by drugs and liposomes across the skin. More sophisticated interpretations of the bilayer mechanics have rendered a more realistic picture of the fate and therapeutic potential of UDV. In this review, we discuss the several mechanisms proposed to explain UDV penetration and provide an update on new preclinical uses of UDL for topical and transdermal drug delivery.

\section{Ultradeformable vesicles}

UDVs are nearly $100 \mathrm{~nm}$ in diameter and are highly deformable (a synonym for elasticity/flexibility) unilamellar vesicles (Figure 1). The first generation of UDVs was made of phospholipids combined with edge activators (EAs). EAs are single-chain surfactants of high radius of curvature and mobility. ${ }^{11}$ In a typical 85:15 (w/w) phospholipids/EA UDV formulation, the EA proportion is close to that required for membrane solubilization, so that the lipid bilayers are destabilized and their deformability is increased. Common EAs are sodium cholate, sodium deoxycholate, Span 80 , Tween 20, Tween 80, and dipotassium glycyrrhizinate. The second generation of Transfersomes was made of at least one bilayer-forming lipid, typically phosphatidylcholine having fluid acyl chains, and at least two more polar lipophilic substances, eg, one surfactant and one surfactantmimicking drug. ${ }^{12,13}$ Two other types of UDVs are the 


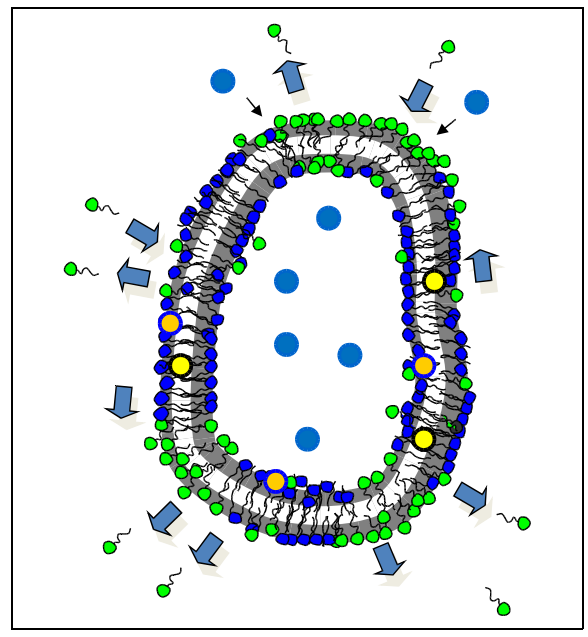

\footnotetext{
Hydrophilic solute $\mathrm{O}$ Hydrophobic solute $\bigcirc$ Amphipathic solute

to Edge activator (EA) \{ Phospholipid or amphypatic bilayer-forming molecule

Mobility in mixing/partitioning/demixing of EA in bilayer $\checkmark$ Partition of hydrophilic solutes
}

Figure I A representative scheme of the lipids and EA comprising UDVs and the carried molecules.

Note: Copyright (C) 2013. Dove Medical Press. Adapted from Romero EL, Morilla MJ. Highly deformable and highly fluid vesicles as potential drug delivery systems: theoretical and practical considerations. Int J Nanomedicine. 2013;8:3।7I-3186. ${ }^{10}$

Abbreviations: EA, edge activator; UDV, ultradeformable vesicle.

nonionic surfactant elastic vesicles and the invasomes. Nonionic surfactant elastic vesicles are made of a bilayerforming surfactant (sucrose laurate ester, L-595) and a micelle-forming surfactant (octaoxyethylene laurate ester, PEG-8-L) acting as EA, plus sulfosuccinate as stabilizer, typically at a 50:50:5 molar ratio. ${ }^{14}$ Invasomes are made of phospholipids such as lysophosphatidylcholine, terpenes (classic permeation enhancer that serves as EA), and ethanol (10\%:0.5\%-1\%:3.3\%, w/v). ${ }^{15}$

The main structural difference between UDVs and conventional liposomes (made of phospholipids with or without cholesterol) is the high and stress-dependent adaptability (high elasticity) of the former versus the bilayer stiffness of the latter. ${ }^{16}$

A generally accepted explanation for bilayer deformability is that as a response to a mechanical stress (such as passing across pores of size smaller than the vesicle diameter), the EAs are demixed from the lipid bilayer and displaced to become relocated in zones of higher curvature/ stress. Phospholipids, on the other hand, enrich the bilayer regions of smaller curvature. Such rearrangements diminish the membrane elastic energy. The average elastic energy of the UDVs is of the same order as the thermal energy $(\kappa \sim k \mathrm{~T})$ and locally may be much smaller than this; for the conventional liposomes, it is known that $\kappa \geq 20 \mathrm{kT}$. $^{7}$ Unlike conventional liposomes, UDVs change shape and volume at minimal energy cost. ${ }^{7,17}$

\section{Methods of preparation and characterization of UDVs}

UDVs are made of phosphatidylcholine plus a pharmaceutically acceptable EA. Tailoring an efficient UDV formulation implies finding the adequate EA type and phospholipid:EA ratio, the starting amount of drugs with suitable physicochemical properties, and the appropriate preparation method. These parameters influence the resulting size, zeta $(Z)$ potential, drug-to-lipid ratio and encapsulation efficiency, elasticity, skin permeation and deposition, and stability.

UDVs can be prepared by the same methods as conventional liposomes such as the film hydration method, solvent injection method, and reverse evaporation technique.

Briefly, in the first method, given amounts of phospholipids and EAs are dissolved in organic solvent and are dried (by evaporation or by spray drying/lyophilization). The resulting lipid film is hydrated in a suitable aqueous buffer under agitation at a temperature nearly $10^{\circ} \mathrm{C}$ above the transition temperature. Multilamellar vesicles in the micrometer-size range are thereafter formed. Hydrophilic drugs dissolved in the hydration medium are trapped in the inner aqueous space. Hydrophobic drugs, which are mixed with lipids in the organic solvent in the first step of preparation, partition into lipid bilayers. The size and lamellarity of the vesicles are reduced by mechanical methods, such as extrusion, sonication, and high-pressure homogenization. 
Typically, the solvent injection method involves the very slow injection of an ethanolic solution of lipids into an aqueous phase, resulting in the formation of unilamellar vesicles. Liposomes obtained in this manner display a well-defined size distribution and high encapsulation efficiency; residual ethanol is then removed by repeated dialysis steps against distilled water. ${ }^{18}$

Reverse phase evaporation involves the formation of an inverse aqueous phase stabilized by phospholipids in an organic solvent such as diethyl ether and isopropyl ether. Subsequent removal of the organic solvent results in unilamellar liposomes with high encapsulation efficiency. ${ }^{19}$

High-pressure homogenization and the solvent injection method are used for liposome preparation on an industrial scale.

Similar to conventional liposomes, the UDV structure depends of four important features: size, zeta potential, lipid concentration, and drug content. Particle mean size, size distribution, and polydispersity are the three most important parameters measured by dynamic light scattering. Microscopic techniques such as transmission electron microscopy, scanning electron microscopy and atomic force microscopy are complementary methods used to determine the morphology and vesicles' lamellarity. Zeta potential is usually measured by phase analysis light scattering and gives information about colloidal stability. Lipids and drug quantification can be performed by spectrophotometric or chromatographic (high-performance liquid chromatography) techniques. Parameters such as drug-to-lipid ratio and encapsulation efficiency are therefore calculated.

In contrast to conventional liposomes, a full structural characterization of UDVs requires determination of their elasticity. Elasticity can be calculated by two methods based on different principles: Van den Bergh method ${ }^{14}$ (rendering a deformability value $D$ ), and the Young's modulus $(E)$.

$D$ is a parameter inversely proportional to $E$ and is calculated as $D=J(r v / r p)^{2}$, where $J$ is the rate of penetration of vesicles through a permeability barrier, $r v$ is the size of vesicles measured after extrusion, and $r p$ is the pore size of the barrier. To measure $J$, vesicles are extruded through two stacked $50 \mathrm{~nm}$ $(r p)$ membranes at $0.8 \mathrm{MPa}$. The extruded volume of vesicles is collected every minute for 15 minutes, phospholipids are quantified in each fraction, and $J$ is calculated as the area under the curve (AUC) of the plot of phospholipids recovered as a function of time. Conventional liposomes would clog up the pores and the extrudate would not contain liposomes. In contrast, UDVs were shown to be able to pass through the pores, suggesting that the latter had flexible membranes. Remarkably, $D$ is inversely proportional to the size of vesicles after crossing the pores. The resulting $D$ is therefore a relative, not an absolute, parameter. As such, it is useful for comparing deformability of different liposomal formulations and to determine whether or not the partitioning of (mostly hydrophobic) drugs into the lipid bilayer would modify the $D$ value.

The Young's modulus $(E)$ or longitudinal elastic modulus is the ratio between the increased tension applied by traction (in the zone of elastic behavior of the material) $(d)$ and the resultant increased relative deformation $(d \varepsilon)(E: d \sigma / d \varepsilon)$. $E$ is a measure of a material's rigidity: the higher the Young's modulus, the stiffer is the material. $D$ and $E$, thus, are inversely related.

Atomic force microscopy (AFM) is a well-suited technique for studying the structural features of liposomes. It enables the simultaneous analysis of the shape and mechanical properties of the bilayers in the nanoscale. The technique allows picking up surface topographical images with a space resolution close to $1 \AA$ and force versus distance curves with a detection limit close to $10^{-12} \mathrm{~N}$. Young's modulus can be obtained from AFM force curves by fitting data with an adequate model of contact. The most popular is the Hertz model: $:{ }^{20} F=2 E \cdot \tan \alpha \cdot \delta^{2} / \pi\left(1-v^{2}\right)$, where $F$ is the force, $E$ is the Young's Modulus, $\alpha$ is the half-opening angle of the conical indenter $\left(53^{\circ}\right.$; based on the geometrical characteristics of the tip and scanning electron microscopy observations), $\delta$ is the indentation, and $v$ is the Poisson radius, which is assumed to be 0.5 for soft biological samples. Using this approach, our research group showed that the values of $E$ for the UDVs were approximately three fold lower than those of conventional liposomes made of soybean phosphatidylcholine $(430 \pm 137 \mathrm{kPa}$ vs $1,119 \pm 242 \mathrm{kPa}$ ) (Higa et al, unpublished data, 2014).

Physicochemical and colloidal stability are also relevant structural parameters of a vesicle population. It is well known that an EA destabilizes and increases the permeability of lipid bilayers. The presence of EAs in vesicles leads to a leakage of the aqueous content of UDVs over time. ${ }^{21}$ Thus, the lack of long-term stability of UDVs is one major drawback.

\section{Mechanism of action}

Two potential mechanisms are currently proposed for the penetration of drugs that are carried by UDVs: 1) a carriermediated mechanism, in which the UDV carries drug molecules into the skin; and 2) a mechanism whereby UDVs act as penetration enhancers; according to this mechanism, vesicles enter the SC and subsequently modify the intercellular lipid 


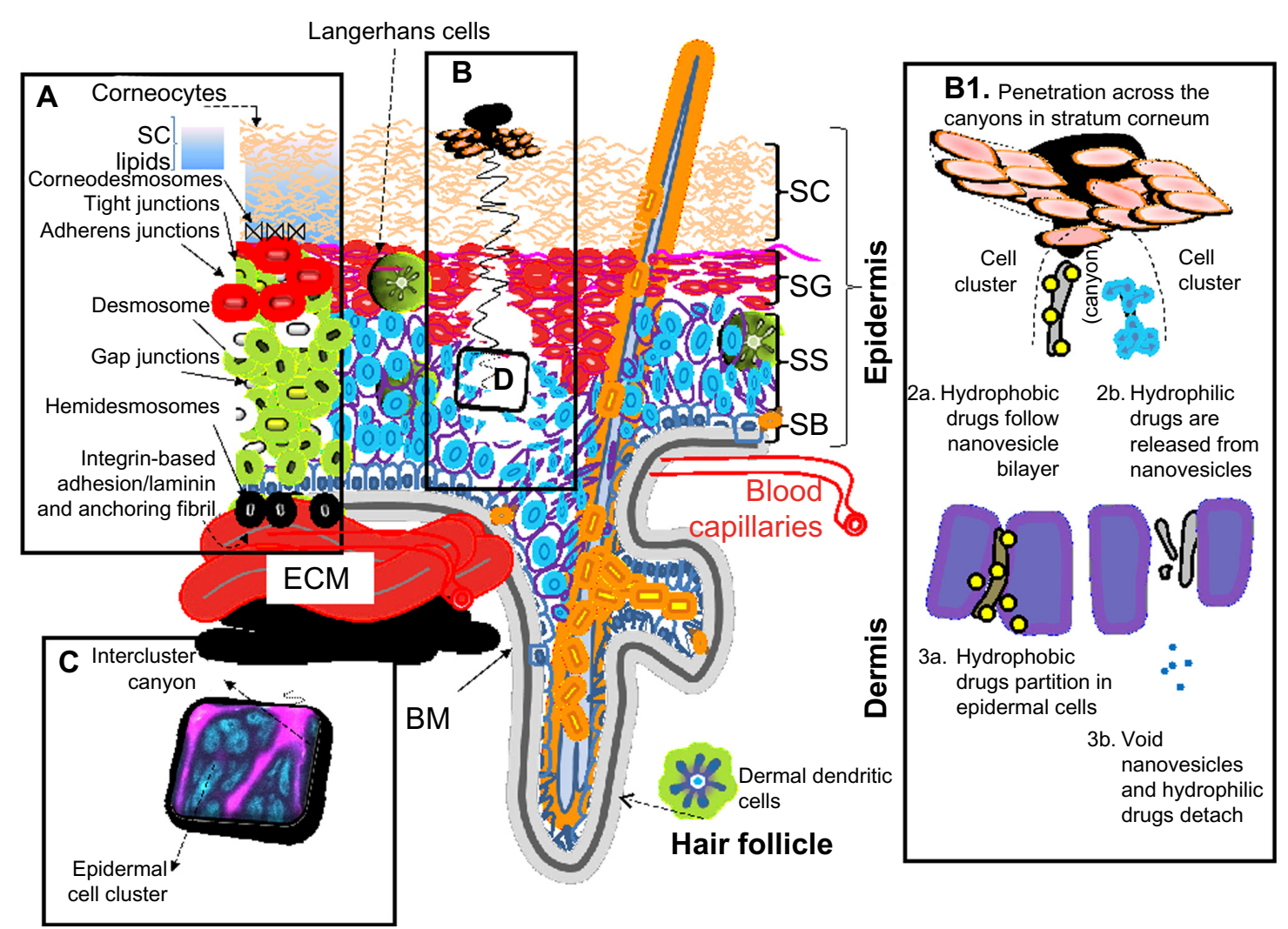

Figure 2 Different cell strata of the epidermis and dermal structures.

Notes: From bottom to top, the strata are as follows. SB (stratum basale): A basal layer containing stem cells (undifferentiated cells that give rise to keratinocytes), keratinocytes (cells that synthesize keratin), and melanocytes (cells that synthesize the pigment melanin that shields DNA from ultraviolet radiation). (A) The different types of intercellular junctions connecting the cells of epidermis. At this level, the cells are bridged by desmosomes (mechanical junctions, involved primarily in cell cohesion; the intracellular ends of desmosomal cadherins form desmosomal plaques, to which keratin filaments bind). The SB is attached to the basal membrane by hemidesmosomes. In the SB, the desmosomes are infrequent and small. SS (stratum spinosum): Several cell layers deep, made of keratinocytes (which become somewhat flattened and "spiny-shaped") and Langerhans (dendritic) cells. The size and number of desmosomes rise significantly. Adherens junctions (intercellular network that coordinates the behavior of a population of cells, coupling intercellular adhesion to the actin cytoskeleton) are present. SG (stratum granulosum): Three to five cell layers deep, made of keratinocytes that accumulate vesicles (granules) filled with the protein keratin and glycolipids, which are exocytosed. The exocytosed keratin wraps around the cell membrane of the keratinocyte, creating a thick coat that provides protection from abrasion and puncture; the exocytosed glycolipids fill the extracellular spaces between the keratinocytes and provides a waterproofing property to skin. The SG forms a barrier between the surface cells and the deeper layers of the epidermis and cuts off nutrient supply to the cells of upper layers. Only at this level, the tight junctions (occluding junctions occurring in simple epithelial and endothelial cells, separating the apical membrane domain from the basolateral part) are found. A reduced number of desmosomes (which are dynamically subjected to degradation by hydrolases), as compared with SS, and adherens junctions are also found. SC (stratum corneum): The SC is a transparent 10-15 $\mu \mathrm{m}$ thick outermost protective dead layer of the skin. It consists of multiple cornified cell layers, the corneocytes (flat dead cells filled with keratin filaments, water, and the natural moisturizing factors), embedded in SC lipids (ceramides, cholesterol, and saturated long-chain free fatty acids, approximately at equimolar ratios. Low levels of other lipid classes are also present, such as cholesterol sulfate, glucosylceramides, and cholesterol esters). The lower SC (SC compactum) contains corneodesmosomes, drastically differing from desmosomes in morphology. The layered structure of the intercellular portion of the junction is lost, whereas the intracellular plaque becomes embedded within the cross-linked cornified envelope. The corneodesmosomes remain functional and their ordinate digestion will eventually permit dissociation of the horny layer and desquamation of superficial corneocytes. The loss of cells from the SC is compensated by the cell growth in the innermost layer of the epidermis, the stratum basale. In this way, the thickness of the epidermis remains approximately constant. (B) A schematic representation of the pathway followed by the UDVs across the canyons of the epidermis; ( $\mathbf{C}$ and $\mathbf{D})$ A confocal fluorescence microscopy image of the epidermis organization in canyons and cell clusters. ${ }^{6}{ }^{8}$ (B I) A schematic representation of the fate of hydrophobic/hydrophilic drugs within UDVs during penetration across the canyons. Reprinted from Colloids Surf B Biointerfaces, I2I. Carrer DC, Higa LH, Defain Tesoriero MV, Morilla MJ, Roncaglia DI, Romero EL. Structural features of ultradeformable archaeosomes for topicaldelivery of ovalbumin. Pages: 28I-289., Copyright 2014, with permission from Elsevier. ${ }^{68}$

Abbreviations: BM, basal membrane; ECM, extracellular matrix; UDVs, ultradeformable vesicles.

lamellae, facilitating the penetration of free drug molecules into and across the SC (Figure 2).

In the first mechanism, the driving force for UDV penetration into the skin is the so-called "xerophobia", the tendency of phospholipids to avoid dry surroundings. ${ }^{22,23}$ To stay fully swollen, UDVs move toward the more hydrated layers of skin (epidermis and dermis), driven by the hydration gradient across the layers (from approximately $10 \%$ to $30 \%$ at the air-exposed skin surface to $75 \%$ in the viable epidermis).
Conventional liposomes dehydrate and fuse in the dry surface of the SC and only accumulate in the upper layers of the SC, not beyond the first micron depth. In contrast, UDVs applied under nonocclusive conditions (because occlusion eliminates the hydration gradient) penetrate the intact skin because of their high deformability. ${ }^{24}$ Hydrophobic dyes carried by UDVs have been detected at several microns' depth below the skin surface, and probably traces of lipid material access the viable epidermis. Despite UDVs being speculated to access 
the blood in the intact form, poorly reproducible results seemed to be achieved. ${ }^{25}$ It was proposed that penetration occurs through hydrophilic intercellular channels of the SC. ${ }^{26}$ Deeper insights on the skin's structural features brought additional controversies related to the idea of xerophobiadriven locomotion from the surface to the deep skin layers. For instance, it was shown that the water gradient across the skin may not be linear and, even in the fully hydrated state, the water content in the lowest SC layers close to the viable epidermis is much lower than that in the central regions of the SC. ${ }^{27}$ According to this observation, deformable vesicles would be stacked within the SC. Moreover, the gradient dissipation beyond the lowest layers of the SC would not be of help for penetration. In addition, hydrophilic pathways on the intact SC cover only a tiny $(<0.001 \%)$ fraction of the normal skin surface and are 10-200 times narrower than the regular size of UDVs $(\sim 100 \mathrm{~nm})$. Thus, UDV movement across these channels would be rather difficult, in spite of their high deformability.

On the other hand, intact UDVs and structural changes in the SC following topical application of UDVs have been visualized within the SC's lipid lamellar regions, but no intact UDVs were detected in the deepest layers of the SC. ${ }^{28}$ Ordinary colocalization analysis of two-color fluorescently labeled liposomes in skin layers, using confocal microscopy, provides substantial information neither about liposome interaction with the skin nor on the persistence of the colloidal structure of liposomes after penetration. Recently, structural tissue features measured at different depths (by multiphoton excitation fluorescence microscopy imaging) combined with stacks of two-dimensional maps of the fluorophore's diffusion coefficient within the tissue (by Raster image correlation spectroscopy) were used to study the skin penetration of UDVs. ${ }^{9}$ These techniques can determine whether two different fluorescent probes (one in the bilayer and the other in the inner aqueous space) diffuse together. A high cross-correlation between the two signals is expected if the liposomes are intact and the hydrophilic content is retained with the vesicle. Both in UDVs and in conventional liposomes, the absence of cross-correlation for the two labels was shown, indicating that below the skin surface, the labels did not diffuse together. This suggested that penetration of intact vesicles is highly compromised by the skin barrier, and that the structural integrity of the vesicles is lost across the SC. The low water activity existing in the SC region may affect the water entropic component (hydrophobic effect), causing the vesicles to lose their structural integrity and hydrophilic content.
On the other hand, recent experimental evidence challenges the parameter elasticity as the paramount factor affecting the penetration of lipophilic and hydrophilic drugs within UDVs. For instance, no differences were registered in terms of the flux of the lipophilic drug, ketoprofen, loaded in nonionic surfactant elastic vesicles or in rigid nondeformable vesicles across the human skin. Moreover, the flux of ketoprofen loaded in UDVs was the lowest and similar to that of the drug solution. ${ }^{29}$ However, the flux of the hydrophilic dye calcein loaded in UDVs and invasomes across the human skin was not linearly correlated with vesicle elasticity. ${ }^{30}$

Regarding the second mechanism, several studies support the fact that surfactants present in UDVs and nonionic surfactant vesicles act as penetration enhancers. ${ }^{31}$ In addition, terpenes of invasomes interact with intercellular lipids, perturbing their lamellar packing and resulting in increased drug solubility in the terpene-treated SC and consequent enhancement in skin penetration.

Overall, the current experimental evidence suggests that the two mechanisms may play a role in the enhanced skin penetration of drugs delivered using UDVs. According to the physicochemical properties of the drug, one of the two mechanisms might predominate. Hydrophilic drugs, for instance, would penetrate driven by the second mechanism, while hydrophobic drugs would use the first one. The penetration of hydrophilic drugs is limited by their partitioning in the lipophilic SC. Therefore, the penetration-enhancing effect seems to play a more important role for hydrophilic, than for lipophilic, drugs (as occurring with many penetration enhancers). Hydrophilic drugs, therefore, might not need to be entrapped in vesicles to enable penetration. Instead, the penetration of hydrophobic drugs is limited by their partition between the SC and the epidermis, which is less lipophilic. In this case, optimal skin penetration is achieved with drugs loaded within vesicles. ${ }^{31}$ Closely related to this subject is the mechanism of drug release from vesicles. Entrapment might result in slow transdermal flux and suboptimum permeation. Once released, drugs' diffusion in skin must occur; thus, the fate of the released drug is dependent on its solubility in the tissue.

The dose per area plays a relevant role in the penetration depth of the carried drug: while a low dose per area favors drug retention in the skin, an increased total applied drug dose and an increased dose per area were shown to promote systemic drug availability. ${ }^{32}$

Summarizing, factors such as drug's electric charge and hydrophilic/hydrophobic balance, drug loading, vesicle elasticity, mode of application (occlusive or nonocclusive), and dosage 
strongly influence the drug's disposition and distribution across the skin. However, physicochemical and pharmacokinetic studies are still needed to define the precise effect of these factors and unravel the mode of action of UDVs.

In the following sections, we provide an overview of the latest studies using UDVs for topical/transdermal drug delivery.

\section{Delivery of anti-inflammatory drugs to musculoskeletal-articular targets}

Nonsteroidal anti-inflammatory drugs (NSAIDs) inhibit the biosynthesis of prostanoids, a family of bioactive lipids (prostaglandins and thromboxane) that play important roles in many cellular responses and pathophysiologic processes. Prostanoids are involved in inflammatory reaction and its resolution, erosion of cartilage and the juxta-articular bone, GI cytoprotection and ulceration, angiogenesis and cancer, hemostasis and thrombosis, renal hemodynamics and progression of kidney disease, and atheroprotection and progression of atherosclerosis. ${ }^{33}$ Prostanoids are generated by the cyclooxygenases COX-1 and COX-2. All NSAIDs are COX-2 inhibitors with some degree of COX-1 inhibition. NSAIDs are generally orally administered and cause mucosal injury throughout the GI tract, as side effects of COX-1 inhibition. Their use is associated with nearly four fold increased risk of serious upper GI complications. This risk is dose dependent but disappears completely approximately 2 months after treatment withdrawal.

Despite topical NSAIDs being used for chronic musculoskeletal pain in adults, the only indication is for chronic pain caused by osteoarthritis. Osteoarthritis is the most common form of joint disease and the leading cause of pain and physical disability in the elderly. Osteoarthritis is characterized by focal areas of loss of articular cartilage in synovial joints accompanied by subchondral bone changes, osteophyte formation at the joint margins, thickening of the joint capsule, and mild synovitis. ${ }^{34}$

Most NSAID molecules diffuse across the SC and into deeper skin regions rather well, through the intercellular lipid matrix of the skin, because of their suitable lipid-water partition coefficient and low MW. ${ }^{35,36}$ However, classic topical formulations (cream and gel) have limited local action because of the fast clearance from the skin by the capillary plexus underlying the skin surface, which leaves too little of the drug in the target organs. ${ }^{35,36}$ Moreover, cardiovascular toxicity has emerged as a previously unrecognized, mechanism-based effect of COX-2 inhibitors when used in high doses in chronic treatments. ${ }^{37}$
The first preclinical study assessing the performance of NSAIDs in Transfersomes was published in 2001 by Cevc and Blume. ${ }^{38}$ Transfersomes enable modification of the route of administration, reduction of the therapeutic dose, and achievement of regioselective delivery to deep subcutaneous tissue of the poorly hydrosoluble NSAIDs diclofenac and ketoprofen (Transfenac ${ }^{\circledR}$ and Diractin ${ }^{\circledR}$, respectively, from IDEA AG, Muenchen, Germany). Transfenac ${ }^{\circledR}$ showed longer effect and reached ten times higher concentrations in the tissues under the skin of mice, rats, and pigs, as compared with diclofenac in a commercial hydrogel. The authors suggested that the drug within the UDVs is cleared less efficiently by the dermal capillary plexus than the free drug. Such an effect was ascribed to the size of the UDVs $(\sim 100 \mathrm{~nm})$, considered too bulky to penetrate through the nonfenestrated capillary of the skin. It was observed, on the other hand, that the AUC for Diractin ${ }^{\circledR}$ gel in the peripheral deep muscle exceeds the AUC for a conventional gel by $\sim 35$ fold. ${ }^{39,40}$ Two Phase III clinical studies showed that Diractin $^{\circledR}$ was superior to oral placebo for the treatment of osteoarthritis of the knee and was comparable with an oral NSAID. ${ }^{11}$

Topical delivery of the poorly hydrosoluble NSAIDs piroxicam, lornoxicam, and diclofenac, as well as the hydrophilic drug ketorolac, using UDVs is less advanced; the results were published between 2011 and 2014. Studies shown in Table 1 include formulation optimization, ex vivo permeation, and only one report of anti-inflammatory activity.

Piroxicam is a nonselective inhibitor of COX with analgesic and anti-inflammatory activity, associated with a number of GI disorders. Piroxicam is used for the treatment of rheumatoid arthritis and osteoarthritis, and it is also used as a potent analgesic. The anti-inflammatory activity of piroxicam in UDVs formulated in a carbopol gel $(0.75 \%$, w/w) was tested on a carrageenan-induced paw edema model. ${ }^{41}$ The piroxicam-UDV gel formulation increased drug permeation by three fold and showed better anti-inflammatory activity, as compared with the free drug in the gel. The piroxicam-UDV gel, however, was topically applied 1 hour before paw edema was induced, making the extrapolation of its activity on a preestablished inflammation difficult. Moreover, the blood levels of the drug were not quantified. On the other hand, the carrageenan-induced inflammation in the rat paw represents a classic model of edema formation and hyperalgesia following subcutaneous injection, arising from proinflammatory agents generated in situ or by infiltrating neutrophils that generate proinflammatory cytokines. ${ }^{42}$ This model however, 
Table I Anti-inflammatory, antihypertensive, and anti-infective drugs incorporated in UDVs

\begin{tabular}{|c|c|c|c|}
\hline Type of drug and properties & Vesicle composition & Vesicle properties & Results \\
\hline \multicolumn{4}{|c|}{ Anti-inflammatory drugs against osteoarthritis } \\
\hline $\begin{array}{l}\text { Piroxicam }{ }^{4} \text { : } \\
\text { lipophilic, } 331.3 \mathrm{Da}\end{array}$ & $\begin{array}{l}\text { SPC:NaDchol, } \\
40 \mathrm{mg}: 2 \% \mathrm{w} / \mathrm{v}\end{array}$ & $\begin{array}{l}\mathrm{I} 50 \mathrm{~nm} \\
-22.7 \mathrm{mV}\end{array}$ & $\begin{array}{l}\text { Cumulative permeation was } 52 \% \text { for UDVs after } 24 \text { hours, with } \\
\text { a flux of } 14.48 \mu \mathrm{g} / \mathrm{cm}^{2} / \mathrm{h} \text { vs } 7.43 \mu \mathrm{g} / \mathrm{cm}^{2} / \mathrm{h} \text { for the free drug in } \\
\text { porcine ear skin ex vivo. } \\
\text { UDVs and the free drug produced } 74 \% \text { and } 60 \% \text { inhibition of } \\
\text { edema, respectively, after } 24 \text { hours, in carrageenan-induced } \\
\text { paw edema model }\end{array}$ \\
\hline $\begin{array}{l}\text { Lornoxicam }{ }^{43}: \\
\text { lipophilic, } 371.8 \mathrm{Da}\end{array}$ & $\begin{array}{l}\text { SPC:chol:T80, } \\
\text { I00:7.74:2.5 w/w/w }\end{array}$ & $\begin{array}{l}\text { EE: } 35 \% \\
280 \mathrm{~nm} \\
16 \mathrm{mV}\end{array}$ & Ex vivo permeation on dorsal skin of rabbit \\
\hline $\begin{array}{l}\text { Diclofenac and } \text { curcumin }^{44} \text { : } \\
\text { lipophilic, } 369.3 \mathrm{Da}\end{array}$ & $\begin{array}{l}\text { SPC: } \mathrm{NaChol,} 3: \text { I molar } \\
\text { ratio, diclofenac and } \\
\text { curcumin were added to } \\
\text { organic solvents }\end{array}$ & $\begin{array}{l}\text { EE: } 98 \%-95 \% \\
67 \mathrm{~nm}\end{array}$ & $\begin{array}{l}\text { Cumulative amount of diclofenac and curcumin in UDVs } \\
\text { was } 37.94 \mu \mathrm{g} / \mathrm{cm}^{2} \text { and } 737.78 \mu \mathrm{g} / \mathrm{cm}^{2} \text {, respectively, with } \\
\text { corresponding fluxes of } 1.58 \mathrm{mg} / \mathrm{cm}^{2} \cdot \mathrm{h} \text { and } 30.74 \mu \mathrm{g} / \mathrm{cm}^{2} \cdot \mathrm{h} \text {, } \\
\text { respectively, on rat skin ex vivo }\end{array}$ \\
\hline \multirow[t]{2}{*}{$\begin{array}{l}\text { Ketorolac trometha mine }{ }^{45} \text { : } \\
\text { hydrophilic, } 255.3 \mathrm{Da}\end{array}$} & $\begin{array}{l}\text { SPC:T80, } 86: 14 \mathrm{w} / \mathrm{w} \\
7 \% \mathrm{v} / \mathrm{v} \text { ethanol }\end{array}$ & EE: $73 \%$ & $\begin{array}{l}\text { UDVs showed long lag time ( } 10 \text { hours), only } \sim 7.7 \% \text { permeated } \\
\text { after } 24 \text { hours on pig's ear skin ex vivo }\end{array}$ \\
\hline & solution of drug & $\begin{array}{l}128 \mathrm{~nm},-12 \mathrm{mV} \\
18.6 \mu \mathrm{g} \mathrm{drug} / \mathrm{mg} \mathrm{SPC}\end{array}$ & $\begin{array}{l}\text { Total amount of ketorolac permeated was } 7-26 \mu \mathrm{g} \text { with a } \\
\text { penetration distance of } 6-33 \mu \mathrm{m} \text { on healthy human volunteers }\end{array}$ \\
\hline
\end{tabular}

Anti-inflammatory drugs against atopic dermatitis Tacrolimus ${ }^{50}$ : SPC:T80, 60:18 w/w lipophilic, 822.9 Da

EE: $70 \%-83 \%$

$123 \mathrm{~nm}$

Glycyrrhetic acid ${ }^{51}$ : lipophilic, $470.6 \mathrm{Da}$

Cetirizine ${ }^{52}$ : lipophilic, $388.8 \mathrm{Da}$

SPC:SA:Span 80, I50:15:16.6 w/w/w

EE: $76 \%$

$140 \mathrm{~nm}$

$12.73 \mathrm{mV}$

\section{Antihypertensive drug \\ Valsartan ${ }^{53}$ : \\ lipophilic, $435.5 \mathrm{Da}$}

$\begin{array}{ll}\text { SPC:NaDchol, } & \text { EE: } 73 \% \\ \text { 3.5:0.62 w/w, } & 87 \mathrm{~nm} \\ \text { 10\% v/v ethanol } & -42.5 \mathrm{mV}\end{array}$

Felodipine ${ }^{54}$

lipophilic, 384.2 Da
SPC:NaDchol, $\quad$ EE: $86 \%$

$85: 15 \mathrm{w} / \mathrm{w}$

$130 \mathrm{~nm}$

EE: $85.14 \%$

$76 \mathrm{~nm}$

$-49.8 \mathrm{mV}$
Cumulative amount of drug was $6.66 \mathrm{mg} / \mathrm{cm}^{2}$ and $4.22 \mathrm{mg} / \mathrm{cm}^{2}$ for UDVs and CLs, respectively, on rat skin layers ex vivo.

Drug was not detected in the fluid collected

On rat skin in vivo, UDVs, CLs, and ointment displayed same distributions of drug in the SC. In epidermis and dermis, amounts of drug in UDVs were 3.8 and 4.2 times as ointment, respectively; in CLs, the amount was only 1.7 times and I.4 times that of ointment; treatment (twice a day for 7 days) with UDVs in gel reduced ear swelling to the minimum, as compared with CLs and ointment

Cumulative amount of drug was $6.94 \mu \mathrm{g} / \mathrm{cm}^{2}$ and $1.30 \mu \mathrm{g} / \mathrm{cm}^{2}$ for UDVs and CLs, respectively, on abdominal rat skin ex vivo. Concentration of drug increased immediately after UDV application and reached $C_{\max }$ at 3 hours on mice skin in vivo. Drug was not detected in plasma. Treatment (twice per day every 3 days $\times 21$ days on ear I hour before and 12 hours after application of DNFB) produced $25.5 \%$ and $34.8 \%$ inhibition of ear thickness for UDVs and for positive control, respectively Skin retention of drug was in the following order: UDV in gel (16.9\%) $>$ liposomal gel $(7.6 \%)>$ cream (3.7\%) on skin of mice ex vivo Treatment (once every alternate day for 2 weeks after half an hour of oxazolone administration) with UDVs in gel significantly decreased itching score and erythema, as compared with cream-treated mice

UDVs showed maximum flux over CLs $\left(626.6 \mu \mathrm{g} / \mathrm{cm}^{2} / \mathrm{h}\right.$ vs $18.5 \mu \mathrm{g} / \mathrm{cm}^{2} / \mathrm{h}$ ) on abdominal skin of Wistar rats ex vivo Oral valsartan controlled the hypertension at 3 hours, then blood pressure rose gradually up to 48 hours. UDVs gradually decreased blood pressure, with the maximum effect observed at 6 hours, and controlled the blood pressure for up to 48 hours UDVs showed highest cumulative drug permeation (94.9\%), skin deposition (8.1\%), and transdermal flux $\left(23.7 \mu \mathrm{g} / \mathrm{h} / \mathrm{cm}^{2}\right)$ on rat skin ex vivo. The maximal permeation from UDVs in gel was 2.6 times higher as compared with free drug gel at 24 hours $T_{\text {max }}$ for topical UDV was 6hours and $C_{\text {max }}$ was $8.05 \mathrm{ng} / \mathrm{ml}, T_{\text {max }}$ for oral drug was 4.85 hours and $C_{\text {max }}$ was $2.31 \mathrm{ng} / \mathrm{ml}$, in rats. $A_{U C C_{0-\infty}}$ was 162.26 and $45.27 \mathrm{ng} \cdot \mathrm{h} / \mathrm{mL}$ for UDVs in gel and oral formulation, respectively 
Table I (Continued)

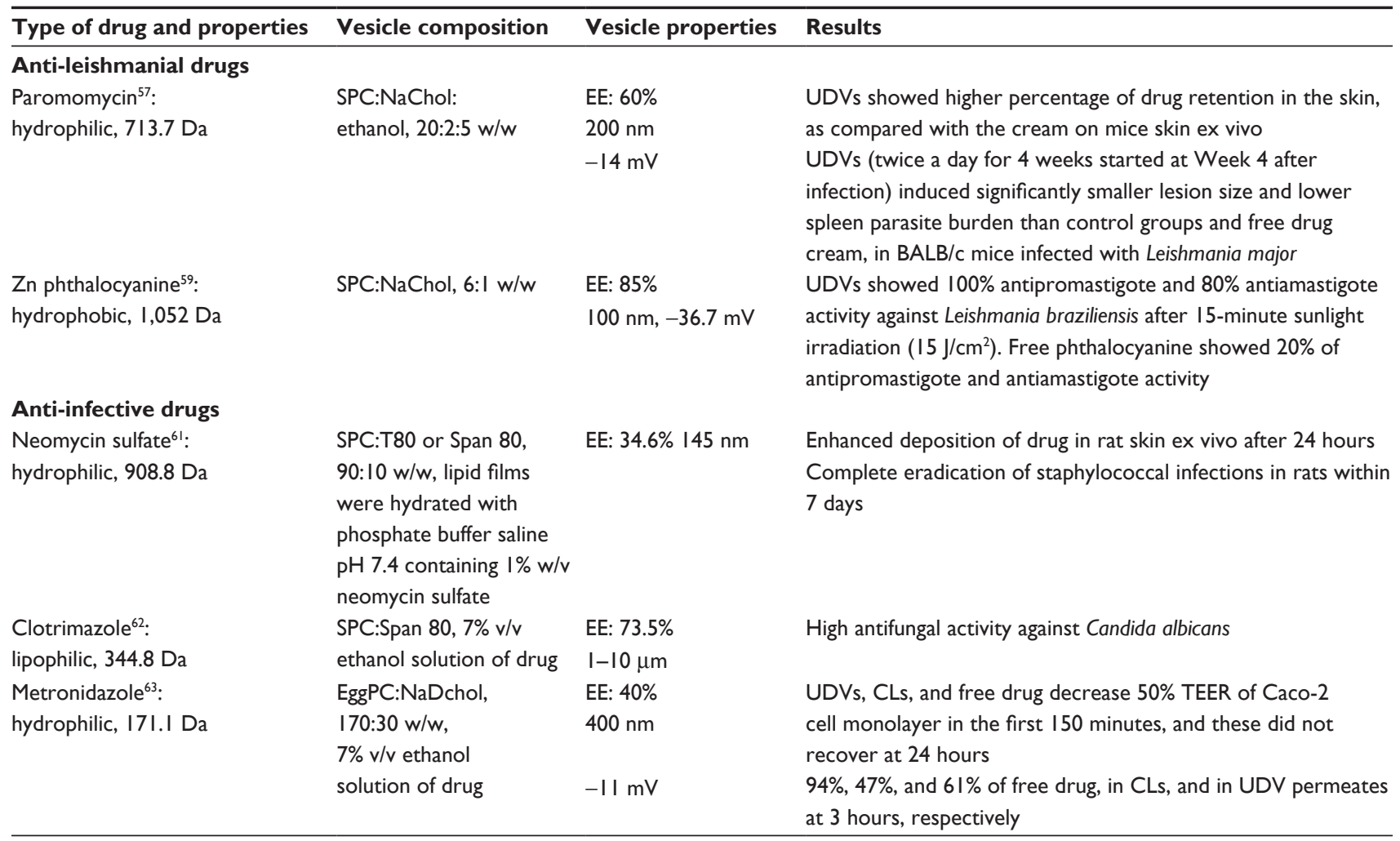

Abbreviations: AUC, area under the curve; chol, cholesterol; $C_{m a x}$, maximum (or peak) serum concentration; $C L$, conventional liposomes; DNFB, 2,4-dinitrofluorobenzene; EggPC, phosphatidylcholine from egg yolk; EE, encapsulation efficiency; NaChol, sodium cholate; NaDchol, sodium deoxycholate; SC, stratum corneum; SPC, soybean phosphatidylcholine; SA, stearylamine; TEER, transepithelial electrical resistance; $T_{\max }$, the time at which $C_{\max }$ is observed; T80, Tween 80 ; UDV, ultradeformable vesicle.

is far from reproducing the anatomopathological context of osteoarthritis or rheumatoid artritis, mainly because of the absence of a capsular barrier.

Lornoxicam is also a potent inhibitor of COX with analgesic, anti-inflammatory, and antipyretic activities, used for the treatment of osteoarthritis and other inflammations. A Box-Behnken model was used to optimize a formulation of lornoxicam in UDVs. ${ }^{43}$ Results only showed that UDVs enhanced lornoxicam permeation through rabbit skin in relation to conventional liposomes.

Diclofenac diethylamine is widely used in arthritis for the symptomatic relief of pain and inflammation. Diclofenac diethylamine is used at low oral doses (25-50 mg), shows poor bioavailability $(40 \%-60 \%)$, and has a short biological half-life (2-3 hours). On the other hand, curcumin, a hydrophobic polyphenol, is a herbal anti-inflammatory agent. Curcumin is orally administered (3.6-12 g/day) and shows poor oral bioavailability, short biological half-life (1.7 hours), and extensive first-pass metabolism. A formulation of diclofenac and curcumin in UDVs was recently developed. ${ }^{44}$ UDVs were reported to deliver nearly 1.4 -fold higher amounts of diclofenac and curcumin across the rat skin, as compared with free drugs. Unfortunately, no data on in vivo activity was included.
Ketorolac tromethamine is a nonselective COX inhibitor used as an analgesic in the management of moderate-tosevere pain, including postoperative pain and visceral pain associated with cancer. Ketorolac shows good oral bioavailability (90\%) with little first-pass hepatic metabolism. Its major drawback is its short biological half-life (4-6 hours), thereby necessitating repeated administrations to achieve a therapeutic effect. Frequent intake of ketorolac leads to severe GI side effects. A formulation of ketorolac in UDVs was recently developed, and the ex vivo permeation on pig's skin and in vivo transdermal delivery in humans were tested. ${ }^{45}$ The ex vivo permeation of ketorolac in UDVs was very low. In vivo, a good correlation between the total amount of permeated drug and the penetration distance versus individual transepidermal water loss was found. These results suggested that the penetration mechanism of UDVs followed the transcutaneous hydration gradient. Although the conditions of the ex vivo and in vivo tests made comparison difficult, in vivo flux was six fold greater than that through excised pig skin. These differences were ascribed to the ex vivo experimental conditions, wherein a long incubation led to dehydration and film formation of the UDV. This hindered UDV penetration and further drug release. Additionally, it was shown than the size of the vesicles remained unchanged upon storage at $4^{\circ} \mathrm{C}$ 
for 1 year, and the registered inner content decreased $52 \%$ within 11 weeks. The most important feature of this work was the use of human volunteers for penetration studies. The in vivo anti-inflammatory activity, as well as the effect and biodistribution after repeated doses over a period of 8-12 weeks (to resemble topical treatments of chronic pain conditions), nonetheless, remain to be tested.

The delivery of NSAIDs to the skeletal muscle requires local drug retention and blood avoidance. Taken together, the above data showed an in vitro high drug penetration, when formulated in UDVs. The drug concentrations in the muscle and blood, however, were not determined. Osteoarthritis, on the other hand, is a pathology wherein conventional formulations would act by reducing the symptoms arising from periarticular structures, partly locally and partly by intracapsular access of drug delivered to blood. The superiority of UDVs for drug delivery against this disease remains to be shown.

\section{Delivery of anti-inflammatory drugs to target layers of the skin against atopic dermatitis}

Atopic dermatitis is a chronic inflammatory skin disease associated with cutaneous hyperreactivity to environmental triggers that are innocuous to normal nonatopic individuals. Atopic dermatitis is a delayed-type hypersensitivity reaction mediated by hapten-specific T cells. Characteristic features include barrier dysfunction, secretion of immunoglobulin E (IgE), epithelial cell hyperplasia, fibrosis, infiltration of inflammatory cells into the dermis and epidermis, and secretion of TH2 cytokines. The major clinical symptoms of atopic dermatitis are pruritic and chronic eczematous skin lesions that are distinguished by infiltration of inflammatory cells. Anti-inflammatory treatment based on topical glucocorticoids and topical calcineurin antagonists (tacrolimus and pimecrolimus) is used for the management of exacerbation and, more recently, for proactive therapy in selected cases ${ }^{46}$ However, the prolonged use of glucocorticoids at high doses causes a variety of systemic and local adverse effects, such as atrophy and telangiectasia, at the skin level. If systemic anti-inflammatory treatment is an option for severe refractory cases, the microbial colonization and superinfection may induce disease exacerbation. In these cases, additional antimicrobia/antiseptic treatment is justified. Systemic antihistamines (H1) can relieve pruritus but do not have sufficient effect on eczema.

Between 1997 and 2004, Cevc and Blume $e^{32,47,48}$ performed a series of studies on mice to assess the performance of topical glucocorticoids in UDVs. It was determined that at low dose per area, the skin toxicity was reduced and sub-SC deposits of corticoids were formed in the epidermis, minimizing the systemic spillover. Hydrophilic drugs such as hydrocortisone could leave the UDVs and diffuse into the surrounding hydrophilic environment, whereas liposoluble triamcinolone acetonide remained associated with UDV bilayers, favoring the formation of an epidermal deposit. ${ }^{32}$ Similar results were achieved in humans by triamcinolone acetonide in UDV s. ${ }^{49} \mathrm{By}$ the accumulation of sub-SC deposits, UDVs proved to increase the performance of low-biological-potency drugs, such as the low-potency hydrocortisone and dexamethasone. ${ }^{48}$

Between 2012 and 2013, tacrolimus, glycyrrhetinic acid, and cetirizine were loaded in UDVs and their activities were tested on chronic allergic contact dermatitis murine models (Table 1).

Tacrolimus is an immunosuppressant macrolide that restrains activation of T-cells and controls the release of proinflammatory cytokines. Tacrolimus blocks cytokine generation and release by basophils, eosinophils, and mast cells, decreasing the number of inflammatory dendritic epidermal cells in the injured layers in atopic dermatitis. A formulation of tacrolimus in UDVs was recently developed and its activity tested on a 2,4-dinitrofluorobenzene (DNFB)induced dermatitis model, using commercial tacrolimus ointment (Protopic) as positive control. ${ }^{50}$ Tacrolimus in UDV srepared with Tween 80 as the EA showed the highest deformability and tacrolimus acummulation in rat skin layers in vitro. UDVs formulated in a $1 \% \mathrm{w} / \mathrm{w}$ carbopol gel showed the highest penetration of tacrolimus in rat epidermis and dermis as compared with Protopic and conventional liposomal gel. In this case, the UDV gel displayed the best therapeutic effects, by depressing ear swelling to the minimum, faster than conventional liposomal gel and Protopic.

Glycyrrhetinic acid, one of the main constituents of the Glycyrrhiza glabra root, is a natural product with antiinflammatory, antiallergic, and interferon-inducing actions but poor bioavailability. The activity of glycyrrhetinic acid in UDVs was tested on a DNFB-induced dermatitis model using triamcinolone acetonide and econazole nitrate commercial cream as positive control. ${ }^{51}$ UDVs were able to reduce the erythema rapidly (suppressing not only the increased ear thickness but also epidermal hyperplasia). The suppressive activity was comparable to that of positive control. Conventional topical triamcinolone acetonide, however, causes intense skin atrophy, a serious side effect that limits its use for chronic skin diseases.

Cetirizine is the active carboxylic acid metabolite of hydroxyzine, a second-generation antihistamine with antiinflammatory properties and high specific affinity for histamine $\mathrm{H} 1$ receptors. Cetirizine is effective in the treatment 
of skin inflammatory conditions. It reduces histamine, bradykinin, and allergen-induced wheal and flare reactions; decreases monocyte and $\mathrm{T}$ lymphocyte chemotaxis, and eosinophil responses. It also decreases intercellular adhesion molecule-1 expression on epithelial cells. However, the oral administration of cetirizine is commonly related to different side effects, including sedation, ocular dryness, tiredness, and dry mouth. A topical formulation is expected to effectively counteract the locally released histamine and other inflammatory mediators. However, no topical formulation of cetirizine is available in the market. Recently, the activity of cetirizine in UDVs was tested on an oxazolone-induced dermatitis model.$^{52}$ UDV s prepared with Span 80 as the EA showed the highest cetirizine entrapment efficiency. UDVs formulated in a $1 \% \mathrm{w} / \mathrm{w}$ carbopol gel showed the best therapeutic effects (reduced the number of eosinophils in dermal tissue, as well as the itching and the erythema scores, in mice), as compared with a conventional cream formulation of cetirizine.

These three lipophilic drugs have to act locally, neither entering the blood nor accessing the underlying skeletal muscle. Taken together, the above data showed a higher skin penetration of the drug and the local in vivo efficacy of drugs in UDVs, as compared with commercial creams. However, none of these experimental approaches quantified the drug in blood and muscle after repeated applications. Further studies are needed to assess the realistic therapeutic usefulness of UDVs in humans.

\section{Delivery of antihypertensive drugs to the target blood circulation}

Hypertension is a chronic disease, with a high chance of causing death, which requires oral long-term treatment with antihypertensive drugs. A transdermal delivery patch of the antihypertensive drug, clonidine, has already been marketed. However, only antihypertensive drugs capable of permeating the skin can be delivered by the transdermal route.

Two lipophilic low-MW drugs, valsartan and felodipine, were recently loaded in UDVs and the activity and pharmacokinetics of these formulations were determined (Table 1).

Valsartan is an antihypertensive drug of low oral bioavailability (25\%) and a mean biological half-life of 7.5 hours. A formulation of valsartan in UDVs was recently developed using a four-factor, three-level Box-Behnken design, and its activity was tested on a rat model of hypertension, induced by methyl prednisolone acetate. ${ }^{53}$ Oral valsartan controlled the hypertension within 2 hours, with the maximum antihypertensive effect observed at 3 hours, but after 3 hours, the blood pressure started rising gradually. Valsartan in UDVs formulated in a carbopol gel showed better antihypertensive activity in comparison with placebo and conventional liposomes. Valsartan in UDVs was released gradually, which resulted in prolonged control of hypertension, with a maximum effect observed at 6 hours and lasting up to 48 hours. As previously reported for the lipophilic corticosteroid triamcinolone acetonide, ${ }^{32}$ an epidermal depot was probably formed, from where the valsartan was released to the blood. Because the heart rate and blood pressure follow a circadian rhythm that increases in the early morning hours and declines in the night, the main challenge of antihypertensive treatments is tuning the drug release to follow such rhythms. In the aforementioned model, the slow release from the depot was sufficient to reduce the hypertension for a period longer than that achieved by the oral route; however, the effect was achieved two fold slower.

Felodipine is a calcium channel antagonist commonly used to treat hypertension and angina pectoris. Felodipine shows extensive hepatic first-pass metabolism, low bioavailability (15\%-20\%), and dose-dependent adverse effect. The pharmacokinetics of felodipine in UDVs was compared with an oral dosage form in Wistar rats. ${ }^{54}$ In vitro permeation of UDVs (prepared with soybean phosphatidylcholine and Span 80) across the rat skin was enhanced in comparison with free drug gel. Topical administration, in contrast to oral delivery, provided relatively constant, sustained blood concentration with minimal plasma fluctuation with rapid and prolonged peak time. The relative bioavailability of felodipine in UDVs was found to be $358 \%$ versus that after oral administration. Stability studies showed that $93 \%$ of the drug was retained after 90 days at $4^{\circ} \mathrm{C}$, with an increase from $75 \mathrm{~nm}$ to $155 \mathrm{~nm}$ in mean size; the impact of this, however, may not be relevant for a topical application. Experimental data are not sufficient to state whether UDVs may improve the antihypertensive effects

\section{Delivery of anti-leishmanial drugs to macrophages of the skin}

Leishmaniasis is a neglected zoonosis caused by the intracellular protozoa of the Leishmania genus, transmitted by the bite of a sandfly vector. Its multiple clinical manifestations include the lethal visceral form, the mostly benign cutaneous form and the more severe and chronic mucocutaneous leishmaniasis.

Once injected into the skin by the bite of sandfly, Leishmania promastigotes invade local phagocytic host cells, wherein the promastigotes transform into amastigotes, which survive in the harsh environment of phagolysosomes. After a brief period during which amastigotes multiply, promastigotes are released in a cell burst, and skin macrophages and dendritic 
cells including Langerhans cells are colonized, together with lymph nodes and mucosa cells. The intracellular location of amastigotes within phagolysosomes is the main structural and phenomenological barrier that leishmanicidal drugs have to overcome. The recommended treatment for cutaneous and mucocutaneous leishmaniasis is the parenteral administration of pentavalent antimonial compounds (sodium stibogluconate or $N$-methylglucamine, daily dose of $20 \mathrm{mg} / \mathrm{kg}$ up to $850 \mathrm{mg} /$ $\mathrm{kg}$ for 3 weeks). ${ }^{55}$ This extensive treatment is sufficient to heal the symptoms of up to $77 \%$ cases but causes significant adverse events. ${ }^{56}$ This drawback, together with the growing resistance to pentavalent antimonials, underscores the need for new therapeutic strategies against cutaneous and mucocutaneous leishmaniasis.

Paromomycin is used in clinical trials for both cutaneous and visceral leishmaniasis since the 1960s. However, its high water solubility and oligosaccharide nature makes its penetration through the SC difficult. Topical application of paromomycin in UDVs was reported to cause a significant reduction in lesion sizes and lowered the parasite burden in spleen in L. majorinfected mice, as compared with paromomycin cream. ${ }^{57}$ In a similar approach, the in vitro drug permeation across stripped skin of paromomycin in large UDVs (300-500 nm) was enhanced and the in vivo activity in L. major-infected mice was improved, as compared with free drug. ${ }^{58}$

Photodynamic therapy (PDT) is an attractive therapeutic alternative that could be used to switch from parenteral to topical administration of leishmanicidal agents. PDT consists of photosensitizers that are excited by light to produce reactive oxygen species, in the presence of oxygen. Because of the accessibility of skin to irradiation from laser or incoherent light sources, PDT has been used with variable outcomes, in experimental and clinical settings against cutaneous leishmaniasis. To increase the delivery of photosensitizer molecules to infected macrophages, recently, the photosensitizer Zn phthalocyanine (ZnPc) was loaded within UDVs. ${ }^{59}$ It was shown that the antiamastigote activity against intracellular L. braziliensis of ZnPc was significantly increased for $\mathrm{ZnPc}$ in UDVs. This was ascribed to the increased penetration into the skin and increased uptake of UDVs by infected cells. Later, the antiamastigote activity of an improved UDV formulation containing total polar lipids extracted from the

Table 2 Other drugs incorporated in UDVs

\begin{tabular}{|c|c|c|c|}
\hline Type of drug and properties & Vesicle composition & Vesicle properties & Results \\
\hline \multicolumn{4}{|l|}{ Anesthetic } \\
\hline Butamben $^{64}$ : lipophilic, 193.2 Da & $\begin{array}{l}\text { EggPC:chol } 4: 3 \\
\text { PEG-8-L at } 2: 3 \\
\text { EggPC molar ratio }\end{array}$ & $\begin{array}{l}\text { EE: } 82.9 \% \\
\text { I50 nm, }-21 \mathrm{mV}\end{array}$ & $\begin{array}{l}\text { UDVs showed maximum flux over conventional liposomes and } \\
\text { free drug }\left(29.93 \mathrm{mg} / \mathrm{cm}^{2} / \mathrm{h}, 23.17 \mathrm{mg} / \mathrm{cm}^{2} / \mathrm{h} \text {, and } 6.95 \mathrm{mg} / \mathrm{cm}^{2} / \mathrm{h} \text {, }\right. \\
\text { respectively) after } 8 \text { hours on full thickness pig ear skin }\end{array}$ \\
\hline \multicolumn{4}{|l|}{ UV protection } \\
\hline \multirow[t]{2}{*}{$\begin{array}{l}\text { Quercetin }{ }^{65} \text { : poorly water } \\
\text { soluble, } 302.2 \mathrm{Da}\end{array}$} & $\begin{array}{l}\text { SPC:chol:Tween } 80 \text {, } \\
4: 1: 1 \mathrm{w} / \mathrm{w} / \mathrm{w}\end{array}$ & EE: $80.41 \%$ & $\begin{array}{l}\text { Penetration rate was } 3.8 \text {-fold greater for UDVs than for drug } \\
\text { suspension. UDVs increased drug deposition in the SC and } \\
\text { epidermis/dermis, as compared with drug suspension }\end{array}$ \\
\hline & & $132 \mathrm{~nm},-21 \mathrm{mV}$ & $\begin{array}{l}\text { UDVs increased cell viability and suppressed reactive oxygen } \\
\text { species in keratinocytes induced by UVB irradiation } \\
\text { Inflammation and collagen fiber breakage were slighter for skin } \\
\text { treated with drug suspension and UDVs in comparison with } \\
\text { control animals, after UVB application. The structure of the } \\
\text { SC was protected and skin edema was relieved. Histological } \\
\text { collagen fibers exhibited no apparent dilatation and congestion } \\
\text { around the capillaries in the UDV-treated group }\end{array}$ \\
\hline \multicolumn{4}{|r|}{ 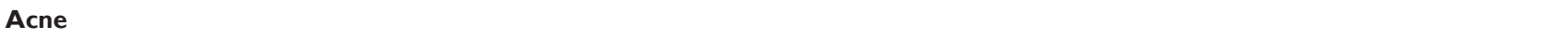 } \\
\hline Tretinoin $^{66}$ : lipophilic, $300.4 \mathrm{Da}$ & PC:Tween 80 & $\begin{array}{l}14.7 \pm 3.0 \mathrm{drug} \\
\text { loading ( } \mu \mathrm{g} / \mathrm{mg} \mathrm{SPC}) \\
131 \mathrm{~nm} \\
5.9 \mathrm{mV}\end{array}$ & $\begin{array}{l}\text { UDVs showed the lowest skin irritation potential after } \\
\text { daily topical application for } 10 \text { days, as compared with } \\
\text { commercial tretinoin formulation (Ketrel). UDV-treated skin } \\
\text { showed irregular hyperkeratosis and presence of fibroblast } \\
\text { proliferation on superficial dermis. For Ketrel-treated skin, } \\
\text { hyperplasia of the epidermis and superficial perivascular } \\
\text { dermatis were observed }\end{array}$ \\
\hline \multicolumn{4}{|l|}{ Osteoporosis } \\
\hline $\begin{array}{l}\text { Raloxifene }{ }^{67}: \text { poorly water } \\
\text { soluble, } 510.5 \mathrm{Da}\end{array}$ & $\begin{array}{l}\text { SPC:NaDchol, } \\
300: 35 \mathrm{w} / \mathrm{w}\end{array}$ & $\begin{array}{l}\text { EE: } 91 \% \\
134 \mathrm{~nm} \\
-2.6 \mathrm{mV}\end{array}$ & $\begin{array}{l}\text { UDVs showed enhancement ratios of } 6.25 \text { for drug } \\
\text { permeation and } 9.25 \text { for skin deposition, respectively, as } \\
\text { compared with conventional liposomes on rat skin }\end{array}$ \\
\hline
\end{tabular}

Abbreviations: chol, cholesterol; CL, conventional liposomes; EggPC, phosphatidylcholine from egg yolk; EE, encapsulation efficiency; NaChol, sodium cholate; NaDchol, sodium deoxycholate; SC, stratum corneum; SPC, soybean phosphatidylcholine; T80, Tween 80; UDV, ultradeformable vesicle; UVB, ultraviolet radiation B. 
hyperhalophile archaea Halorubrum tebenquichense was tested. The new formulation was more extensively taken up by macrophages than UDVs lacking archaeolipids, and it was observed to eliminate intracellular L. braziliensis amastigotes, without reducing the viability of host cells, keratinocytes, and bone marrow-derived dendritic cells, when irradiated at $0.2 \mathrm{~J} / \mathrm{cm}^{2} .{ }^{60}$

\section{Delivery of anti-infective drugs to superficial microbial infections of the skin}

Infections affecting the mucosal surfaces, such as the GI tract, the respiratory and vaginal tracts, and the ocular surface, are ordinarily treated with anti-infective drugs of short residence time at the site of application and poor bioavailability. This leads to incomplete elimination of organisms, recurrence, and tolerance. A minimal penetration depth, together with a prolonged local action (considering, for instance, crossing or attaching to the vaginal mucus layer), avoiding blood delivery is required for topical formulations. Recently, neomycin sulfate, ${ }^{61}$ clotrimazole, ${ }^{62}$ and metronidazole ${ }^{63}$ in UDVs were used to improve the treatment of dermal Staphylococcus aureus infection and vaginal superficial fungal infections (Table 1). Despite a higher drug penetration of clotrimazole or metronidazole in UDVs being shown in vitro, these studies provide no conclusive evidence on the suitability of UDVs for vaginal application.

\section{Delivery of other drugs}

In vitro skin permeation of the local anesthetic butamben, ${ }^{64}$ the antioxidant quercetin, ${ }^{65}$ the anti-acne agent tretinoin, ${ }^{66}$ and the antiosteoporosis raloxifene hydrochloride ${ }^{67}$ in UDVs has recently been reported (Table 2). A higher drug penetration of butamben and raloxifene in UDVs than in conventional liposomes was shown in vitro. Besides, quercetin in UDVs protected mice skin from photodamage caused by ultraviolet radiation $\mathrm{B}$, while absence of in vivo skin irritation was shown for tretinoin in UDVs.

\section{Conclusion}

In this review, we have discussed the performance of a wide range of drugs (mainly lipophilic and low-MW drugs) loaded in UDVs with the intention of enhancing their penetration across the SC and to target diseases at different skin depths. A close analysis of the experimental settings suggests the need for a rational framework to improve the performance of UDVs. Clearly, different sites of the skin such as epidermis/dermis, subcutaneous muscle, or blood circulation could not be successfully targeted using the same formulation. The current experimental evidence suggests that UDV formulations and in vivo dosages should be tailored to accomplish each therapeutic goal. A major difference between parenteral and topical drug delivery is that pharmacokinetics and biodistribution of drugs in UDVs are linked to the chemical structure of the drug. For instance, transdermal drug delivery to access the blood by avoiding the oral route could be achieved with hydrophilic instead of hydrophobic drugs because the former ones are released from UDVs and could diffuse in the intercellular environment of the viable epidermis toward the dermis. Instead, hydrophobic drugs remain associated with the UDV lipid matrix and their chances to leak out toward deeper layers are lower. Subcutaneous muscle, on the other hand, could be targeted by UDVs slowly releasing hydrophobic drugs. Above all, a single structural feature - the high elasticity is insufficient to target sites at different depths (epidermis, dermis, muscle, intra-articular tissue, or blood). Very often, however, a high in vitro drug penetration across rat skin is the single experimental evidence presented as a succeeding proof of concept on depth-specific target. Data of therapeutic levels at the target site and, very importantly, of blood levels of the drug (particularly after repeated applications during extended treatments, to discard the probability of toxicity of drugs having serious systemic side effects) have to complement the in vitro or in vivo penetration assessment, which alone, is insufficient as predictor of therapeutic activity. Exhaustive studies of in vivo drug biodistribution as a function of UDV structure are lacking.

\section{Disclosure}

The authors report no conflicts of interest in this work.

\section{References}

1. Morrow DIJ, McCarron PA, Woolfson AD, Donnelly RF. Innovative strategies for enhancing topical and transdermal drug delivery. Open Drug Delivery J. 2007;1:36-59.

2. Hadgraft J, Guy R, editors. Transdermal Drug Delivery. Developmental Issues and Research Initiatives. New York: Marcel Dekker; 1989.

3. Bouwstra JA, Ponec M. The skin barrier in healthy and diseased state. Biochim Biophys Acta. 2006;1758:2020-2080.

4. Bangham AD, Horne RW. Negative staining of phospholipids and their structured modification by surface active agents as observed in the electron microscope. J Mol Biol. 1964;8:660-668.

5. Weissig V, Pettinger TK, Murdock N. Nanopharmaceuticals (part 1): products on the market. Int J Nanomedicine. 2014;9:4357-4373.

6. Schütz CA, Juillerat-Jeanneret L, Mueller H, Lynch I, Riediker M. Therapeutic nanoparticles in clinics and under clinical evaluation. Nanomedicine. 2013;8:449-467.

7. Cevc G. Material transport across permeability barriers by means of lipid vesicles. In: Lipowsky R, Sackmann E, editors. Handbook of Biological Physics. Vol I. Netherlands: Elsevier Science BV; 1995:465-489. 
8. Cevc G, Schätzlein A, Gebauer D, Blume G. Ultra-high efficiency of drug and peptide transfer through the intact skin by means of novel drug-carriers, transfersomes. In: Bain KR, Hadgkraft J, James WJ, Water KA, editors. Prediction of Percutaneous Penetration. Vol 3b. Cardiff: STS Publishing; 1993:226-234.

9. Brewer J, Bloksgaard M, Kubiak J, Sorensen JA, Bagatolli LA. Spatially resolved two-color diffusion measurements in human skin applied to transdermal liposome penetration. J Invest Dermatol. 2012;133: 1260-1268.

10. Romero EL, Morilla MJ. Highly deformable and highly fluid vesicles as potential drug delivery systems: theoretical and practical considerations. Int J Nanomedicine. 2013;8:3171-3186.

11. Cevc G. Rational design of new product candidates: the next generation of highly deformable bilayer vesicles for noninvasive, targeted therapy. J Control Release. 2012;160:135-146.

12. Cevc G, Vierl U, inventors; Idea AG, assignee. NSAID formulations, based on highly adaptable aggregates, for improved transport through barriers and topical drug delivery. United Sates patent US7473432 B2. January 6, 2009.

13. Cevc G, Vierl U, inventors; Idea AG, assignee. Aggregates with increased deformability, comprising at least three amphipats, for improved transport through semi-permeable barriers and for the noninvasive drug application in vivo, especially through the skin. United Sates patent US20090155235 A1. June 18, 2009.

14. Van den Bergh BAI, Vroom J, Gerritsen H, Junginger HE, Bouwstra JA. Interactions of elastic and rigid vesicles with human skin in vitro: electron microscopy and two- photon excitation microscopy. Biochim Biophys Acta. 1999;1461:155-173.

15. Verma DD. Invasomes-novel topical carriers for enhanced topical delivery: characterization and skin penetration properties, $\mathrm{PhD}$ thesis, Marburg/Lahn, 2002.

16. van den Bergh BAI, Wertz PW, Junginger HE, Bouwstra JA. Elasticity of vesicles assessed by electron spin resonance, electron microscopy and extrusion measurements. Int J Pharm. 2001;217:13-24.

17. Elsayed MM, Cevc G. The vesicle-to-micelle transformation of phospholipid - cholate mixed aggregates: a state of the art analysis including membrane curvature effects. Biochim Biophys Acta. 2011; 1808:140-153.

18. Batzri S, Korn ED. Single bilayer liposomes prepared without sonication. Biochim Biophys Acta. 1973;298:1015-1019.

19. Szoka F Jr, Papahadjopoulos D. Procedure for preparation of liposomes with large internal aqueous space and high capture by reverse-phase evaporation. Proc Natl Acad Sci U S A. 1978;75:4194-4198.

20. Velegol SB, Logan BE. Contributions of bacterial surface polymers, electrostatics, and cell elasticity to the shape of AFM force curves. Langmuir. 2002;18:5256-5262.

21. Montanari J, Roncaglia D, Lado L, Morilla MJ, Romero EL. Avoiding failed reconstitution of ultradeformable liposomes upon dehydration. Int J Pharm. 2009;372:184-190.

22. Warner RR, Lilly NA. Correlation of water content with ultrastructure in the stratum corneum. In: Friedrich-Schiller PE, Berardesca E, Maibach HI, editors. Bioengineering of the Skin. Water and the Stratum Corneum, Volume I. Dermatology: Clinical and Basic Science Part No 6. Boca Raton, FL: Informa Healthcare; 1994:3-12.

23. Cevc G, Blume G. Lipid vesicles penetrate into intact skin owing to the transdermal osmotic gradients and hydration force. Biochim Biophys Acta. 1992;1104:226-232.

24. Cevc G, Gebauer D. Hydration-driven transport of deformable lipid vesicles through fine pores and the skin barrier. Biophys J. 2003;84: $1010-1024$.

25. Cevc G, Schätzlein A, Richardsen H. Ultradeformable lipid vesicles can penetrate the skin and other semi-permeable barriers unfragmented. Evidence from double label CLSM experiments and direct size measurements. Biochim Biophys Acta. 2002;1564:21-30.

26. Schätzlein A, Cevc G. Non-uniform cellular packing of the stratum corneum and permeability barrier function of intact skin: a high-resolution confocal laser scanning microscopy study using highly deformable vesicles (Transfersomes). Br J Dermatol. 1998;138:583-592.
27. Williams A. Transdermal and Topical Drug Delivery. 1st ed. London: Pharmaceutical Press; 2003.

28. Honeywell-Nguyen PL, de Graaff AM, Groenink HW, Bouwstra JA. The in vivo and in vitro interactions of elastic and rigid vesicles with human skin. Biochim Biophys Acta. 2002;1573:130-140.

29. Uchino T, Lefeber F, Gooris G, Bouwstra J. Characterization and skin permeation of ketoprofen-loaded vesicular systems. Eur J Pharm Biopharm. 2014;86:116-156.

30. Ntimenou V, Fahr A, Antimisiaris SG. Elastic vesicles for transdermal drug delivery of hydrophilic drugs: a comparison of important physicochemical characteristics of different vesicle types. J Biomed Nanotechnol. 2012;8:613-623.

31. Elsayed MMA, Abdallah OY, Naggar VF, Khalafallah NM. Lipid vesicles for skin delivery of drugs: reviewing three decades of research. Int J Pharm. 2007;332:1-16.

32. Cevc G, Blume G. Biological activity and characteristics of triamcinolone-acetonide formulated with the self-regulating drug carriers, transfersomes. Biochim Biophys Acta. 2003;1614:156-164.

33. Smyth EM, Grosser T, Wang M, Yu Y, FitzGerald GA. Prostanoids in health and disease. $J$ Lipid Res. 2009;50:S423-S428.

34. Altman R, Asch E, Bloch D, et al. Development of criteria for the classification and reporting of osteoarthritis: classification of osteoarthritis of the knee. Arthritis Rheum. 1986;29(8):1039-1049.

35. Singh P, Roberts MS. Skin permeability and local tissue concentrations of non-steroidal anti-inflammatory drugs after topical application. J Pharmacol Exp Ther. 1994;268:144-151.

36. Hadgraft J, de Plessis J, Goosen C. The selection of non-steroidal antiinflammatory agents for dermal delivery. Int J Pharm. 2000;207:31-37.

37. Patrono C, Rocca B. Nonsteroidal antiinflammatory drugs: past, present and future. Pharmacol Res. 2009;59:285-289.

38. Cevc G, Blume G. New, highly efficient formulation of diclofenac for the topical, transdermal administration in ultradeformable drug carriers, transfersomes. Biochim Biophys Acta. 2001;1514:191-205.

39. Cevc G, Mazgareanu S, Rother M, Vierl U. Occlusion effect on transcutaneous NSAID delivery from conventional and carrier-based formulations. Int J Pharm. 2008;359:190-197.

40. Cevc G, Mazgareanu S, Rother M. Preclinical characterisation of NSAIDs in ultradeformable carriers or conventional topical gels. Int $J$ Pharm. 2008;360:29-39.

41. Shaji J, Lal M. Preparation, optimization and evaluation of transferosomal formulation for enhanced transdermal delivery of a COX-2 inhibitor. Int J Pharm Pharm Sci. 2014;6:467-477.

42. Morris CR. Carrageenan-induced paw edema in the rat and mouse. In: Winyard PG, Willoughby DA, editors. Method in Molecular Biology. Inflammation Protocols. Vol 225. Totowa, NJ: Human Press Inc.; 2003:115-121.

43. Taha EI. Lipid vesicular systems: formulation optimization and ex vivo comparative study. J Mol Liquids. 2014;196:211-216.

44. Chaudhary H, Kohli K, Kumar V. Nano-transfersomes as a novel carrier for transdermal delivery. Int J Pharm. 2013;454:367-380.

45. Nava G, Piñón E, Mendoza L, Mendoza N, Quintanar D, Ganem A. Formulation and in vitro, ex vivo and in vivo evaluation of elastic liposomes for transdermal delivery of ketorolac tromethamine. Pharmaceutics. 2011;3:954-970.

46. Darsow U, Wollenberg A, Simon D, et al. ETFAD/EADV eczema task force 2009 position paper on diagnosis and treatment of atopic dermatitis. JEADV. 2010;24:317-328.

47. Cevc G, Blume G, Schatzlein A. Transfersomes-mediated transepidermal delivery improves the regio- specificity and biological activity of corticosteroids in vivo. J Control Release. 1997;45:211-226.

48. Cevc G, Blume G. Hydrocortisone and dexamethasone in very deformable drug carriers have increased biological potency, prolonged effect, and reduced therapeutic dosage. Biochim Biophys Acta. 2004;1663:61-73.

49. Fesq H, Glöckner D, Abeck D, et al. Improved risk-benefit ratio for a triamcinolone acetonide transfersome formulation in comparison to a commercial triamcinolone acetonide formulation. $\mathrm{Br} J$ Dermatol. 2003;149:611-619. 
50. Lei W, Yu C, Lin H, Zhou X. Development of tacrolimus-loaded transfersomes for deeper skin penetration enhancement and therapeutic effect improvement in vivo. Asian J Pharm Sci. 2013;8:336-345.

51. Li S, Qiu Y, Zhang S, Gao Y. Enhanced transdermal delivery of $18 \beta$-glycyrrhetic acid via elastic vesicles: in vitro and in vivo evaluation. Drug Dev Ind Pharm. 2012;38(7):855-865.

52. Goindi S, Kumar G, Kumar N, Kaur A. Development of novel elastic vesicle-based topical formulation of cetirizine dihydrochloride for treatment of atopic dermatitis. AAPS PharmSciTech. 2013;14:1284-1293.

53. Ahad A, Aqil M, Kohli K, Sultana Y, Mujeeb M, Ali A. Formulation and optimization of nanotransfersomes using experimental design technique for accentuated transdermal delivery of valsartan. Nanomedicine. 2012;8:237-249.

54. Yusuf M, Sharma V, Pathak K. Nanovesicles for transdermal delivery of felodipine: development, characterization, and pharmacokinetics. Int J Pharm Investig. 2014;4:119-130.

55. Tuon FF, Amato VS, Graf ME, Siqueira AM, Nicodemo AC, Amato Neto V. Treatment of new world cutaneous leishmaniasis - a systematic review with a meta-analysis. Int J Dermatol. 2008;47:109-124.

56. Lawn SD, Armstrong M, Chilton D, Whitty CJ. Electrocardiographic and biochemical adverse effects of sodium stibogluconate during treatment of cutaneous and mucosal leishmaniasis among returned travellers. Trans R Soc Trop Med Hyg. 2006;100:264-269.

57. Bavarsad N, Bazzaz BSF, Khamesipour A, Jaafari MR. Colloidal, in vitro and in vivo anti-leishmanial properties of transfersomes containing paromomycin sulfate in susceptible BALB/c mice. Acta Trop. 2012;124:33-41

58. Carneiro G, Santos DC, Oliveira MC, et al. Topical delivery and in vivo antileishmanial activity of paromomycin loaded liposomes for treatment of cutaneous leishmaniasis. J Liposome Res. 2010;20:16-23.

59. Montanari J, Maidana C, Esteva MI, Salomon C, Morilla MJ, Romero EL. Sunlight triggered photodynamic ultradeformable liposomes against Leishmania braziliensis are also leishmanicidal in the dark. J Control Release. 2010;147:368-376.
60. Perez AP, Casasco A, Schilrreff P, et al. Enhanced photodynamic leishmanicidal activity of hydrophobic zinc phthalocyanine within archaeolipids containing liposomes. Int J Nanomedicine. 2014;9: 3335-3345.

61. Darwhekar G, Jain DK, Choudhary A. Elastic liposomes for delivery of neomycin sulphate in deep skin infection. Asian J Pharm Sci. 2012;7: 236-241.

62. Kumar R, Rana AC, Bala R, Seth N. Formulation and evaluation of elastic liposomes of clotrimazole. Int J Drug Dev Res. 2012;4(3):348-355.

63. Vanić Ž, Hafner A, Bego M, Škalko-Basnet N. Characterization of various deformable liposomes with metronidazole. Drug Dev Ind Pharm. 2013;39(3):481-488.

64. Cereda CM, Franz-Montan M, da Silva CM, et al. Transdermal delivery of butamben using elastic and conventional liposomes. J Liposome Res. 2013;23(3):228-234.

65. Liu D, Hub H, Lin Z, et al. Quercetin deformable liposome: preparation and efficacy against ultraviolet $\mathrm{B}$ induced skin damages in vitro and in vivo. J Photochem Photobiol B Biology. 2013;127:8-17.

66. Ascenso A, Salgado A, Euletério C, et al. In vitro and in vivo topical delivery studies of tretinoin-loaded ultradeformable vesicles. Eur $J$ Pharm Biopharm. 2014;88(1):48-55.

67. Mahmood S, Taher M, Mandal UK. Experimental design and optimization of raloxifene hydrochloride loaded nanotransfersomes for transdermal application. Int J Nanomedicine. 2014;9:4331-4346.

68. Carrer DC, Higa LH, Defain Tesoriero MV, Morilla MJ, Roncaglia DI, Romero EL. Structural features of ultradeformable archaeosomes for topicaldelivery of ovalbumin. Colloids Surf B Biointerfaces. 2014;121:281-289.
Research and Reports in Transdermal Drug Delivery

\section{Publish your work in this journal}

Research and Reports in Transdermal Drug Delivery is an international, peer-reviewed, open access online journal publishing original research, study protocols, reviews, editorials and commentaries on all aspects of transdermal drug delivery. Specific topics in the journal include: Laboratory and clinical development of drug delivery systems including preclinical, clinical studies and protocols; Rationale and basic science; Drug

\section{Dovepress}

delivery via gels, creams or patches; Use of chemical drug penetration enhancers Patient acceptability studies; and pharmacoeconomic and clinical outcome studies. The manuscript management system is completely online and includes a very quick and fair peer-review system, which is all easy to use. Visit http://www.dovepress.com/ testimonials.php to read real quotes from published authors. 